\title{
Warfarin Initiation and the Potential Role of Genomic-Guided Dosing
}

\author{
Craig R. Lee, PharmD
}

[See related article pp. 207-213]

\author{
Keywords: \\ Warfarin, \\ Anticoagulation, \\ Pharmacogenetics, \\ Pharmacokinetics, \\ CYP2C9, \\ Adverse events, \\ Multvariate dosing models, \\ Prothrombin time
}

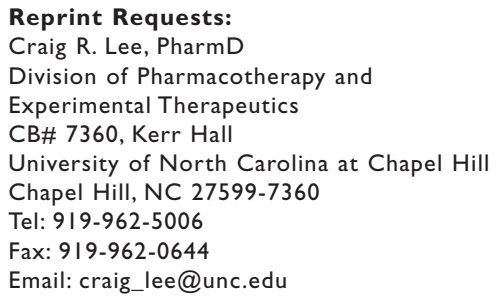


More recently, various retrospective studies have demonstrated that certain genetic polymorphisms in $C Y P 2 C 9$ and $V K O R C 1$ also significantly influence warfarin dose requirement. Higashi and colleagues ${ }^{2}$ demonstrated that patients carrying a variant $C Y P 2 C 9 * 2$ or $* 3$ allele had significantly lower warfarin maintenance dose requirements compared to $C Y P 2 C 9 * 1 / * 1$ (wild type) individuals and were at higher risk of a supratherapeutic INR, required a longer period of time to achieve stable dosing and were significantly more likely to experience a bleeding event during the initiation of therapy. Rieder and colleagues ${ }^{3}$ subsequently demonstrated that genetic variation in VKORC1 also significantly influenced warfarin maintenance dose requirements accounting for a greater proportion of the variability in dose than $C Y P 2 C 9$ genotype. Collectively, the results of these and other investigations suggest that $C Y P 2 C 9$ and $V K O R C 1$ genomic information, in addition to established factors such as age and concomitant medications, may be clinically useful during the initiation of warfarin therapy when the maintenance dose requirement is unknown and patients are at the greatest risk for adverse bleeding events. However, the clinical utility of a genomic-guided dosing strategy remains to be evaluated in large, prospective clinical trials.

Prior to completion of such prospective studies, we first need to: (1) determine the feasibility of obtaining and incorporating genomic information into warfarin dosing nomograms during the initiation of therapy, and (2) better understand the relative contribution of genomic and non-genomic factors to the warfarin dose-response relationship during this initiation period. Studies by Hillman, Wilke and colleagues (Hillman et al. ${ }^{4}$ and Wilke et al. ${ }^{5}$ ) published in Clinical Medicine \& Research have provided important insight into these critical questions.

First, Hillman and colleagues ${ }^{4}$ demonstrated that prospective implementation of a genomic-guided dosing nomogram in patients being initiated on warfarin therapy is feasible, such that both patients and physicians are willing to participate and that the incorporation of genomic information into an individualized dosing strategy can occur in a timely and efficient manner (the average turnaround time from blood collection to genotype determination and dose calculation was approximately 4 hours). Although this single-center pilot study was underpowered to evaluate clinical outcomes such as bleeding events, the findings do suggest that genomic-guided dosing may be superior to standard approaches at predicting a patient's maintenance dose requirement, particularly in individuals with a variant $C Y P 2 C 9$ allele.

Second, using a novel mathematical modeling approach, Wilke and colleagues 5 extensively characterized the influence of various genomic and non-genomic factors on the warfarin dose-INR response relationship during the initial 30 days of therapy. This approach provided important insight into the relative contribution of individual patient factors such as dose, age, comorbidities, concomitant medication use and CYP2C9 genotype on the rate of anticoagulation in a real-world patient population, reinforcing the importance of factors such as age and concomitant medication use on warfarin sensitivity. Moreover, their extensive modeling of this mathematical relationship will undoubtedly improve the accuracy and generalizability of future multivariate dosing nomograms similar to the one utilized in their feasibility study. ${ }^{4}$ Importantly, future studies will be necessary to evaluate the influence of genetic variation in $V K O R C 1$ on this dose-response relationship during the initiation phase of therapy.

The ultimate goals of pharmacogenomics is to account for and to minimize inter-individual variability in drug response, to maximize beneficial and minimize adverse drug effects and, ultimately, to improve clinical outcomes. In time, the aforementioned statement of clinical observation may be revised to: The routine implementation of genomic and clinical data into individualized dosing strategies has accounted for a substantial proportion of inter-patient variability in warfarin sensitivity and has improved outcomes. Although the need remains for additional prospective studies in larger populations that will evaluate the influence of such genomic-guided dosing strategies on the risk of clinical outcomes, such as bleeding events, the investigations by Hillman, Wilke and colleagues 4,5 have taken a critical next step towards potential implementation of such strategies into clinical practice.

\section{References}

1. Ansell J, Hirsh J, Poller L, Bussey H, Jacobson A, Hylek E. The pharmacology and management of the vitamin $\mathrm{K}$ antagonists: the Seventh ACCP Conference on Antithrombotic and Thrombolytic Therapy. Chest 2004;126(3 Suppl):204S-233S.

2. Higashi MK, Veenstra DL, Kondo LM, Wittkowsky AK, Srinouanprachanh SL, Farin FM, Rettie AE. Association between CYP2C9 genetic variants and anticoagulation-related outcomes during warfarin therapy. JAMA 2002;287:1690-1698.

3. Rieder MJ, Reiner AP, Gage BF, Nickerson DA, Eby CS, McLeod HL, Blough DK, Thummel KE, Veenstra DL, Rettie AE. Effect of VKORC1 haplotypes on transcriptional regulation and warfarin dose. N Engl J Med 2005;352:2285-2293.

4. Hillman MA, Wilke RA, Yale SH, Vidaillet HJ, Caldwell MD, Glurich I, Berg RL, Schmelzer J, Burmester JK. A prospective, randomized pilot trial of model-based warfarin dose initiation using CYP2C9 genotype and clinical data. Clin Med Res 2005;3:137-145.

5. Wilke RA, Berg RL, Vidaillet HJ, Caldwell MD, Burmester JK, Hillman MA. Impact of age, CYP2C9 genotype, and concomitant medication on the rate of rise for prothrombin time during the first 30 days of warfarin therapy. Clin Med Res 2005;3:207-213.

\section{Author Affiliation}

Craig R. Lee, PharmD, Division of Pharmacotherapy and Experimental Therapeutics, School of Pharmacy, University of North Carolina at Chapel Hill, Chapel Hill, North Carolina 